\title{
(Re)produire, marquer et (s')approprier des « lieux (publics) de ville " par les mots ou comment les murs (dé)font les langues à Dschang
}

\author{
Jean-Benoît Tsofack*
}

\begin{abstract}
Résumé
Dans cette contribution, nous voulons interroger ce " désordre » linguistique dans une ville camerounaise marquée comme on le sait du sceau du plurilinguisme. Autrement dit, il est question de voir comment les murs (enseignes murales ou publiciaires) y font les langues, c'està-dire, construisent des pratiques linguistiques hétérogènes, mais aussi comment ces mêmes murs défont les langues, les modifient de manière « inconsciente et permanente », ou, à contrario, comment les langues font ou défont les murs. On est donc ainsi amené à constater que l'espace public est aussi un «espace discursif » et le lieu même du « désordre » linguistique où ce qui est important, ce n'est pas ce que les gens parlent ou écrivent, mais comment ils parlent et écrivent, communiquent « dans ce désordre, ou malgré ce désordre, voire même grâce à ce désordre ", comment les langues ont "mise en scène » et s'affichent publiquement.

Nous partons de l'observation indirecte des pratiques linguistiques faite sur un corpus non sollicité d'énoncés publicitaires (toponymes, enseignes diverses) recueillis lors d'une enquête de terrain menée en 2007 dans la ville de Dschang. Cette méthodologie relève dans l'ensemble, de l'Analyse du Discours telle que l'ont pensée Bulot et Veschambre (2006b), c'est-à-dire, une analyse du «procès d'appropriation de l'espace », de son «marquage » langagier.
\end{abstract}

\begin{abstract}
In this contribution, we examine the linguistic 'disorder' in a Cameroonian city marked by multilingualism. In other words, we look into how city walls (signs or advertisements) make languages, i.e. how
\end{abstract}

\footnotetext{
* Université de Dschang. E-mail : tsofackb@yahoo.fr
} 
they construct heterogeneous linguistic practices, but also how those same walls unmake languages, by changing them 'unconsciously and permanently' or, a contrario, how languages make or unmake walls. We therefore observe that the public space is also a 'discursive space' and the very locus of the linguistic 'disorder' where what is important is not what people say or write, but rather how they speak and write, how they communicate 'in this disorder, or despite this disorder, or even through this disorder', how languages are publicly 'staged' and displayed.

Based on indirect 'observation' of linguistic practices carried out, we used an 'unsolicited' corpus of advertising discourse (various signs and place names) gathered during a field survey conducted in 2007 in the city of Dschang. Our overall methodology pertains to Discourse Analysis as conceived by Bulot and Veschambre (2006b), i.e. an analysis of the 'process of appropriation of space' and its linguistic 'marking'.

\section{Introduction}

La sociolinguistique urbaine envisage l'espace de ville comme une entité doublement articulée révélant les dimensions multiples de sa prise de sens. L'espace est d'abord l'aire matérielle, c'est-à-dire un espace social qui rend compte des rapports complexes entre lien social et langues ou pratiques langagières. Il est ensuite lieu, c’est-à-dire un fait matériel nommé, isolable et localisé par rapport aux autres, démarqué par des repères et identifié en discours comme étant inscrit dans un système autonome qui rend compte de l'organisation socio-spatiale de l'espace considéré (Bulot 2002). Les êtres humains, en effet, produisent bien des signes sur la surface terrestre pour signaler une appropriation ou leur présence, et ceux-ci ont bien des significations qui parfois dévoilent des motivations et des intentions, mais aussi des pratiques linguistiques. Autrement dit, le lieu affiche non seulement sa «mise en mots », c'est-à-dire ses caractéristiques identificatoires, sa signification ou son programme "praxémique », mais aussi ses pratiques et ses représentations linguistiques à travers ces mêmes signes. Ceux-ci peuvent être intentionnels et, dans ce cas, ils sont « destinés à signaler l'appropriation » de l'espace ou du lieu (Bulot et Veschambre 2006).

Cela revient à dire que l'espace ou le « lieu de ville », qu'il soit privé ou public, est, au-delà de sa réalité matérielle, une production du ou des discours qui contribuent à le façonner et à lui donner sens. Sa mise en mots constitue non plus seulement une forme de "marquage " ou d' " appropriation " linguistique, langagière ou identitaire par la communauté sociale, mais aussi un indice permettant de renseigner sur ses pratiques linguistiques, lesquelles pratiques, dans un environnement plurilingue comme celui du Cameroun, participent de l' « hétérogénéité » (Blanchet 2007), et, dans une certaine mesure, du « désordre et de la complexité » (Calvet 2007). 
L'occasion est ainsi donnée lors de ce colloque d'interroger ce « désordre » linguistique dans la ville de Dschang, et plus particulièrement dans l'un de ses multiples lieux publics caractéristiques qu'est l'entrée principale de l'Université. Il sera question de voir comment les murs (enseignes murales ou publicitaires) y font les langues, c'est-à-dire construisent des pratiques linguistiques, mais aussi comment ces mêmes murs défont les langues, les modifient de manière "inconsciente et permanente ", ou, a contrario, comment les langues y font ou défont les murs. On est, de ce fait, amené à constater que l'espace public est aussi un « espace discursif » (Bulot 2001) et le lieu même du « désordre » linguistique où ce qui est important, ce n'est pas ce que les gens disent ou écrivent, mais comment ils parlent et écrivent, communiquent « dans ce désordre, ou malgré ce désordre, voire même grâce à ce désordre » (Calvet 2007), comment les langues affichent publiquement leur propre «mise en scène ».

En nous fondant sur la distinction théorique in vitro / in vivo (Calvet 1994), nous avons observé indirectement les pratiques linguistiques dans cet espace public à travers un corpus non sollicité d'enseignes diverses (toponymiques, publicitaires...) recueilli lors d'une enquête de terrain menée en juillet-août 2008. La méthodologie relève, dans l'ensemble, de l'Analyse du Discours telle que l'ont pensée Bulot et Veschambre (2006), c'est-à-dire une analyse du « procès d'appropriation de l'espace » et de son « marquage » langagier. Cette approche qui privilégie la significativité des faits à leur représentativité devrait nous permettre de mettre en lumière le fait que l'espace public en ville peut être construit, produit ou même administré par les discours des acteurs, mais qu'il est aussi un espace d'affichage, voire de « mise en scène » des langues et des discours qui participent dans l'ensemble, de la complexité des situations linguistiques au Cameroun.

La communication commencera par expliciter son cadre théorique et son terrain de recherche, ensuite elle examinera les paradigmes de dénomination, l'affichage des langues et enfin elle questionnera le poids des langues nationales dans la sphère publique.

\section{Des « lieux (publics) de ville » comme textes}

La sociolinguistique urbaine s'est référée à la ville de deux façons différentes, en étudiant les paroles dans et sur la ville en général ou une portion de la ville en particulier. C'est donc dire que le rôle des discours est central dans la perception, l'organisation, la configuration, voire l'administration (linguistique) de la ville qui se veut un espace aux dimensions multiples. Son traitement peut ainsi relever d'une approche qui considère qu'elle est donnée à voir, à déchiffrer, à consommer publiquement, qu'elle est dense de signes à lire et que le rapport que les usagers citadins établissent avec elle est un 
rapport de « décodage, de lecture interprétative, voire de consommation spectaculaire » (Mondala 2000:31). La lecture des signes, des symboles et des textes parsemés dans l'espace urbain devient ainsi l'objet d'une analyse signifiante, d'où l'intérêt porté à ce qui est inscrit, écrit, codé, lu, décodé dans l'espace, le territoire, le paysage. La cité, dira d'ailleurs Roland Barthes (1985:265), est un discours, et ce discours est véritablement un langage. La ville « est une écriture : celui qui se déplace dans la ville, c'est-à-dire l'usager de la ville (ce que nous sommes tous), est une sorte de lecteur qui, selon ses obligations et ses déplacements, prélève des fragments de l'énoncé pour les actualiser en secret ». L'expression "langage de la ville », qui a fort opportunément fait l'objet d'un colloque international et pluridisciplinaire à Albi (France) en 2002, ${ }^{1}$ est en lui-même polysémique, et peut renvoyer soit aux usagers qui y parlent (la ville est alors un cadre d'activité), soit à la ville elle-même (la ville est alors un acteur anthropomorphisé) (Mondala op. cit. 32-33).

Le premier aspect de cette conception que nous abordons pour cette communication met en avant la nécessité d'interroger les discours qui circulent dans la ville et qui font la ville, non plus métaphoriquement, mais performativement, dans diverses situations de paroles et de pratiques des langues caractéristiques de la pratique sociale. La ville, en effet, est un espace d'hétérogénéités qui pose de façon emblématique la question de la variation, du changement, du contact de langues, à propos d'identités et de pratiques socio-langagières multiples et hybrides. Elle est devenue le lieu idéal pour observer et questionner la " complexité » sociale des pratiques langagières, la dynamique du changement linguistique, les formes et les effets du contact entre les langues différentes.

\section{De l'hétérogénéité des pratiques linguistiques}

Louis-Jean Cavet (in Moreau 1997:179-180) distingue dans l'histoire des langues ou des rapports entre des langues deux grands mouvements. L'un, lié à la pratique sociale des locuteurs (ce qu'ils font avec les langues, comment elles sont réellement parlées, etc.) qui, dans leur usage au quotidien, " interviennent sur la langue et sur les langues », en modifient les formes et les situations de manière " inconsciente et permanente ", qu'on appelle action in vivo. L'autre, lié à l'action de l'Etat ou de la politique linguistique, voire des planificateurs, est le fruit d'une intervention « consciente, raisonnée, ponctuelle » appelée action in vitro. Cela revient à dire qu'il y a, de manière générale, deux façons d'intervenir sur les langues et sur les situations sociolinguistiques : d'une part, l'une qui procède des politiques linguistiques et des pratiques institutionnelles, et, d'autre part, l'autre qui procède de la pratique sociale qui est, pour reprendre Didier de Robillard (2001), loin d'être 
« prédictible ». Car, s'il est vrai que les langues n’existent pas sans les gens qui les parlent (Calvet 1993:3), on peut comprendre que toute situation linguistique est le produit «sans cesse mouvant » d'un «rapport homéostatique », d'un système de régulation entre les besoins linguistiques de la société et les fonctions sociales des langues, c'est-à-dire les réponses sociales que pouvaient apporter les langues aux demandes linguistiques de la société (Calvet cité par Gasquet-Cyrus 2008:92). On ne peut ainsi que constater le « désordre » dans lequel les gens ${ }^{2}$ communiquent. ${ }^{3}$ Il ne s'agit pas seulement des gens qui parlent pour produire des énoncés « destinés à alimenter la machine bien huilée du linguiste », mais des gens « qui parlent au quotidien, des gens qui discourent, qui interpellent, qui racontent, qui chantent, qui rient, qui jurent, qui crient, qui exagèrent, qui jargonnent, qui inventent des formes, qui écrivent sur des panneaux, des affiches, des murs... » (Ibidem). Ce qui est constitutif des langues, en effet, ce n'est ni leur matérialité, ni leur structure, mais la croyance dans leur existence, ancrée dans le besoin qu'elles existent (de Robillard 2005:145).

\section{Du terrain et du corpus}

De ce point de vue, Dschang, localité lovée sur les hautes terres de l'ouest du Cameroun, est certes une " petite » ville, mais avec un destin immense, une ville au grand cœur, comme on pourrait le dire. Révélée en effet depuis 1895 par l'Allemand Zintgraff lors de la deuxième expédition militaire allemande au Cameroun, ce n'est qu'en 1903, date de sa refondation par la mission militaire impériale allemande, que la ville a fait l'objet d'un suivi historique. Depuis lors, elle a connu trois tutelles européennes successives : allemande de 1895 à 1916, puis anglaise de 1916 à 1920 et française enfin jusqu'à l'indépendance en 1960. D’un poids géopolitique et économique enviable, la ville a vu sa population, son influence et son prestige s'accroître assez lentement pendant plusieurs années, avant d'exploser subitement à partir de 1993 lorsque, au terme d'une réforme universitaire (Décret présidentiel n 92/264 du 29 décembre 1992), ce qui était jusque-là le "Centre universitaire de Dschang ${ }^{4}$ fut érigé en même temps que trois autres centres universitaires, ${ }^{5}$ en université d'Etat. Cette explosion démographique soudaine a donné un nouveau visage à la ville, tant les migrations internes ont connu une ampleur sans précédent. La population est ainsi passée de près de 100000 habitants en 1967 à plus de 400000 habitants en 2003. ${ }^{6}$ Notons qu'avant 1990 la population de la ville était constituée majoritairement de natifs et à la limite des communautés des villes et départements voisins, mais avec l'avènement de l'institution universitaire, elle est devenue plus hétérogène et plus métissée, tant toutes les composantes socioculturelles ou sociolinguistiques du pays y sont 
représentées. C'est donc dire le rôle de l'université dans la configuration sociale, spatiale et même (socio) linguistique ${ }^{7}$ de la nouvelle ville et le poids que peuvent y avoir non seulement les langues, mais aussi les discours sur la façon dont les «lieux de ville », ou, dans une large mesure, la spatialité urbaine sont configurées, administrées et même appropriées par les divers acteurs de la vie sociale.

En effet, la croissance démographique observée a non seulement modifié le visage (socio) linguistique de la ville, mais aussi provoqué de profondes mutations économiques, sociales et administratives qui ont créé et généré tant des situations de discours et de pratiques linguistiques dans la mosaïque des supports observés et observables. L'augmentation considérable des recettes municipales, le développement du petit commerce, l'inauguration et l'ouverture de la route Dschang-Melon (qui relie la ville à la province du Littoral), la création d'un commissariat central (de police)... sont sans doute les effets positifs et les paradigmes de l'urbanisation qui ont accompagné la création de l'université et la mutation globale du visage et de l'espace urbain. De nouveaux secteurs d'activités ont jailli, que ce soit en termes de transport urbain (pour faciliter les déplacements dans la ville) ou en termes de petits commerces (bars, discothèques, secrétariats bureautiques, cybercafés, restaurants...) partout dans la ville ou aux alentours des grands espaces publics, à l'exemple de l'entrée principale de l'université. Chacun de ces commerces (ces lieux) a pour souci de marquer « en langue » sa présence, de signifier à travers des inscriptions murales ou publicitaires diverses sa raison d'être. C'est justement à ce type de discours que nous avons décidé de porter notre attention, en nous interrogeant sur la façon dont les langues et les murs sont « mis en scène », sur le poids des langues dans la signalétique des espaces et des lieux (publics).

\section{Les paradigmes publics de (de)nomination}

La notion de marquage est centrale en sociolinguistique urbaine et en géographie sociale. Elle se réfère à la « manière de signaler une appropriation de l'espace » (Ripoll 2006:17. Le marquage représente toujours une action matérielle, soit à travers la fabrication, la réutilisation des repères signifiants, soit à travers la présence des corps ou des signes dont ils sont porteurs, et des langues ou parlures qu'ils pratiquent. Le marquage peut être dit « signalétique » lorsqu'il se réfère à « l'ensemble des traces, des marques qui permettent à un individu de s'orienter dans l'espace social/ sociolinguistique (Bulot et Veschambre 2006). De ce point de vue, on peut sérier le marquage linguistique de l'espace public à l'entrée de l'université de Dschang de deux manières : d'une part, un marquage signalétique à visée publicitaire qui a pour simple rôle de signaler une présence (enseignes 
publicitaires, affiches de reconnaissance), et, d'autre part, un marquage identitaire qui va au-delà de la simple présence pour signaler une modalité d'appropriation de l'espace ou encore une matérialisation de l'identité individuelle ou collective. C'est le cas dans l'ensemble des enseignes publicitaires qui parsèment cet espace, avec, pour rôle, d'attirer l'attention de la clientèle sur l'existence du lieu et la fonction qu'il occupe dans l'espace considéré. Nous avons ainsi répertorié une mosaïque d’activités qui contribuent à matérialiser et à administrer linguistiquement l'espace, et cette administration procède, comme nous le verrons plus tard, elle aussi d'un certain « désordre ». On y distingue :

Les restaurants : 4 au total, dont deux portent le nom générique de « restaurant », ou l'une de ses variantes apocopiques « restau », suivi d'un caractérisant adjectival « merveille » (1a) ou un caractérisant d'appartenance (identitaire), c'est-à-dire le nom du propriétaire (« Mme Coach » (1b) :

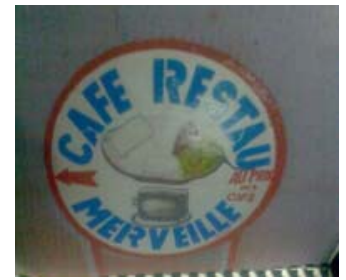

(1a)

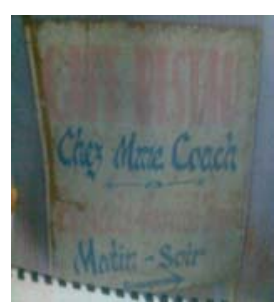

(1b)

Ces dénominations génériques rendent la référence du lieu assez explicite, et l'usager n'éprouve aucune difficulté à se repérer dans l'espace ou pour repérer le lieu-dit. Mais l'enseigne (1c) est assez caractéristique, avec une référence de type métonymique où le lieu est désigné génériquement par l'un des produits qu'on y retrouve, le « café ». On pourrait a priori penser qu'il s'agit d'un simple café, mais dans la réalité du lieu, il s'agit d'un restaurant où on retrouve non seulement à manger, mais aussi à boire, du café par exemple (petit-déjeuner), ce que confirme d'ailleurs l'enseigne (1b) qui est plus explicite sur son référent publicitaire («Café-Restau») . L'enseigne (1d) est lui aussi caractéristique, puisqu'elle n’affiche d'emblée aucun paradigme de référenciation explicite, mais plutôt le nom du (de la) propriétaire. Ce qui y est mis en avant, ce n'est pas le caractère publicitaire de la référence (et donc l'activité), mais c'est l'indexicalisation de la personne ou la focalisation sur l'identité (le nom) du propriétaire:

(1c) : CHRISTAL COFFEE CENTER : Arôme-Vitalité-Saveur. Dégustez et appréciez l'arôme originel et la force ancestrale. 
(1d) : BIENVENUE CHEZ MAÁ-THÉ.

Menu du jour...

Déjeuner complet

Riz + sauce tomate + sauce d'arachide

Couscous manioc + sauce de gombo (sauce pistache)

Couscous maïs + dolé

Macabo + dolé

Taro + sauce jaune

Condré

Les photocopies sont au nombre de 7 et portent dans l'ensemble la dénomination générique de "photocopie », qui est assez explicite sur le référent. La seule différence réside au niveau du caractérisant qui accompagne la mention générique à chaque fois. Cette caractérisation est, dans certains cas, nominale et déterminative («Photocopie de $\mathrm{N} »$ ) :

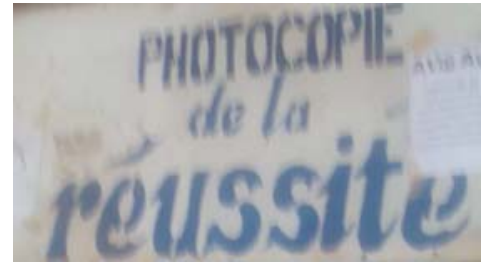

(2a)

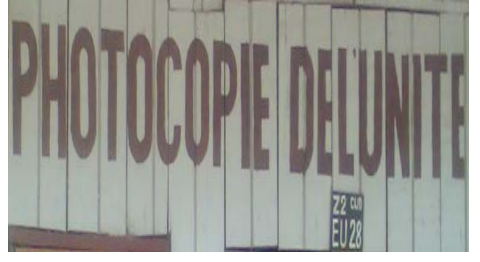

(2b)

Dans d'autres cas, elle est nominale et identitaire («Photocopie $\mathrm{N} »)$ ), avec la présence d'un nom de personne ou d'un toponyme (un lieu assez célèbre comme une ville par exemple) :

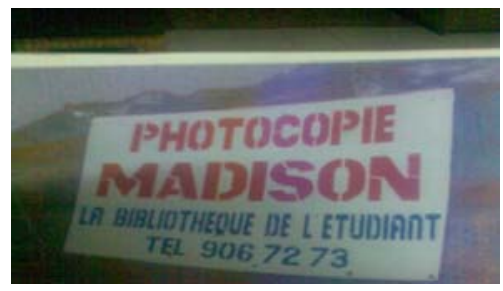

(2c) 
Dans d'autres cas encore, elle est anonyme: l'enseigne ne comporte que le dénominatif générique explicite (« Photocopie ») .

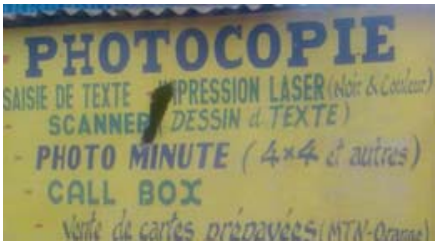

$(2 d)$

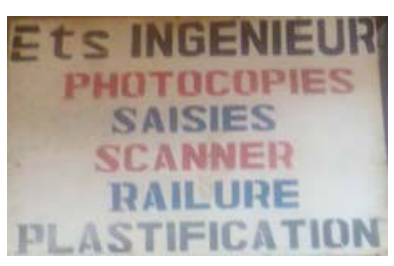

(2e)

Les secrétariats-bureautiques sont les plus nombreux (13 au total) et les plus représentés dans cet espace public, avec des paradigmes de dénomination variés. On peut en distinguer quatre grands paradigmes, avec à chaque fois un lexème générique dominant :

Le premier paradigme est celui où dominent les désignatifs « Centre de formation » « Multiservices », avec plus ou moins la mention explicite de l'activité du lieu et plus ou moins un caractérisant postposé ou antéposé (lequel caractérisant peut être nominal ou adjectival) :

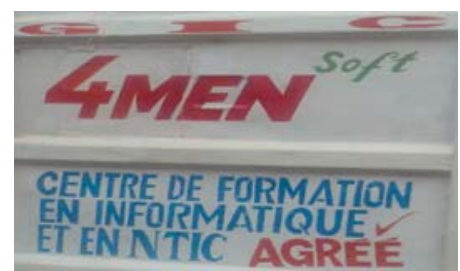

(3a)

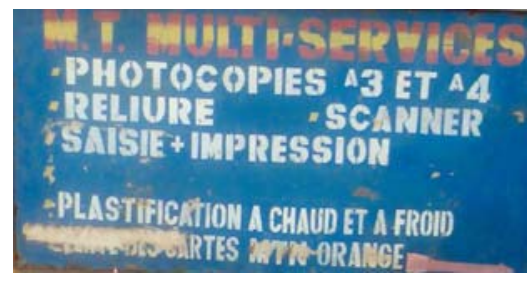

(3b)

Le second paradigme est celui où dominent les lexèmes génériques «Bureautique » «Business » (5), avec plus ou moins un caractérisant exprimé, antéposé ou postposé :

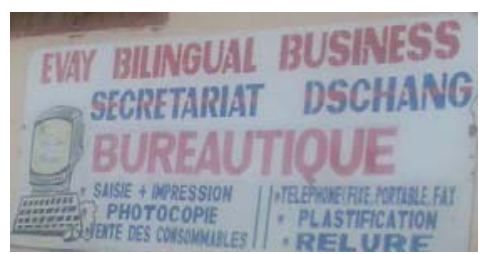

(3c)

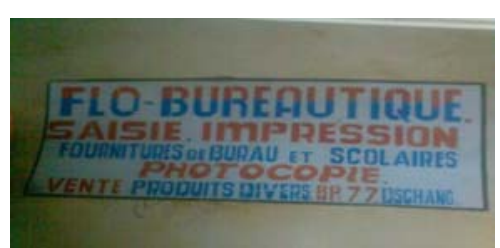

(3d) 
- Le troisième paradigme est celui où dominent les lexèmes « Centre » / «Center » (en français et en anglais), avec, lui aussi, un caractérisant adjectival ou nominal postposé ou antéposé :

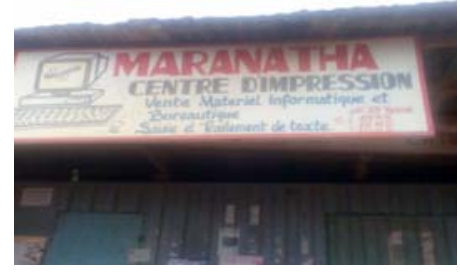

(3e)

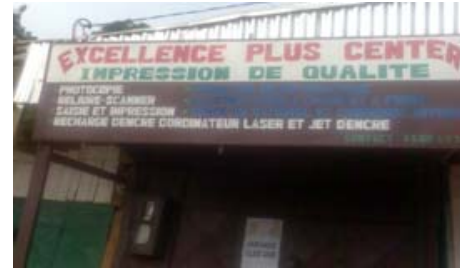

(3f)

Le dernier paradigme est celui où dominent des lexèmes tels que «Info » / « Digital » Computer » qui renvoient tous à l'univers informatique, ce qui sera explicité par les descriptions des activités contenues à la suite de l'enseigne :

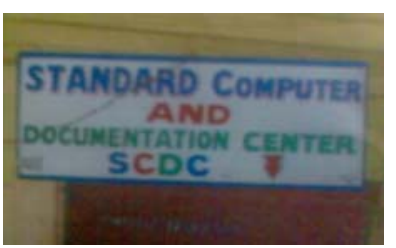

(3g)

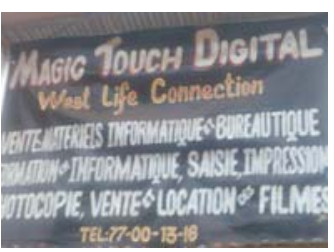

(3h)

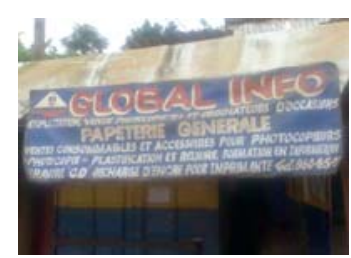

(3i)

Les cyber-espaces sont au nombre de deux, avec des enseignes où domine la désignation générique de « Cyber », avec la présence plus ou moins explicite d'un caractérisant adjectival ou nominal:

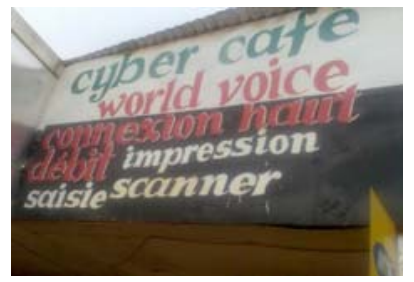

(4)

D’autres types d'activités sont aussi représentés dans cet espace et les modes de désignation procèdent du même principe, à savoir un nom générique renvoyant à l'activité, accompagné d'un caractérisant (nominal ou adjectival). 
On dénombre ainsi une boutique pour vêtements, un studio photo numérique, une structure de micro-finance, une herboristerie chinoise et un centre artisanal. Mais ce qui est impressionnant dans l'ensemble, c'est le cas des dénominations mixtes où se cumulent dans une même enseigne plusieurs désignatifs renvoyant à deux activités différentes dans le même espace : photocopie + secrétariat ; photocopie + prêt à porter, photocopie + secrétariat + photo + call box, ${ }^{8} \ldots$

Dans l'ensemble, la tendance dans l'administration et l'appropriation de cet espace public est à l'hétérogénéité, à l'imprévisibilité, à l'instabilité (et donc ... au " désordre »). Hétérogénéité dans la mosaïque des activités qui meublent cet espace réduit, hétérogénéité dans le jeu des couleurs dans la fabrication des enseignes, et hétérogénéité enfin dans l'organisation linguistique des enseignes elles-mêmes. Il n’y a pas un modèle unique de présentation, mais des modèles qui caricaturent autant de goûts, de désirs, de perceptions et de compétences linguistiques, culturelles ou intellectuelles des référents dont l'université et la ville en général sont porteuses. Il n’y a pas non plus de norme unique dans la disposition linguistique des enseignes (le formel côtoie l'informel, l'anonyme l'explicite et le non-dit le dit), les langues et les énoncés standards côtoient habilement des énoncés approximatifs et des langues qu'on peut caractériser de «mixtes ». Si nous prenons juste quelques exemples, en (2e) et en (3h), on verra que les enseignes portent des constructions approximatives de « Railure » au lieu de «reliure » (2e), de «Filmes » au lieu de « films », etc., ce qui donne la preuve de la présence dans cet espace de compétences et de normes linguistiques plurielles, malgré la proximité avec l'université qui est le lieu par excellence de la préservation et du conditionnement de la norme.

Un autre exemple caractéristique de cette hétérogénéité des normes et des compétences est révélé par cette enseigne d'un salon de coiffure où l'on peut observer une méprise sur les termes "pédicule » (au lieu de " pédicure »), «manicule » au lieu de «manucure »).

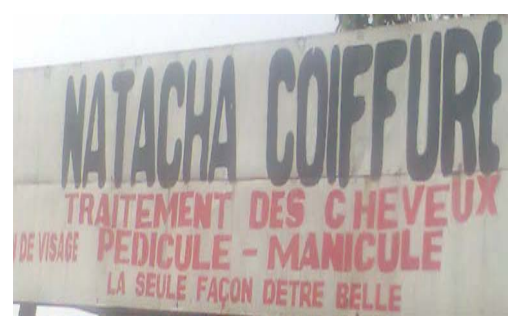

(5) 
Ce « désordre » est donc symptomatique de ce que peuvent être les langues et les pratiques linguistiques dans un espace public non normalisé ou codifié, et pour ne pas dire, un espace hétérogène, ce qui nous amène donc à dire que si l'on peut prévoir dans une situation de plurilinguisme ce que l'on peut parler (ou écrire) dans une situation formelle, on ne saurait prévoir (prédire) ce que les locuteurs écriraient ou diraient et comment ils le diraient dans des situations non formelles. Les pratiques linguistiques et les besoins sociaux qui les conditionnent s'autorégulent en ville, un endroit qui est un lieu par excellence de brassage linguistique, mais aussi et en même temps un lieu où ce plurilinguisme va se résoudre, évoluer, dessinant par là même « les formes futures de cohabitation entre les locuteurs de ces différentes langues » (Calvet 1994:73). Une analyse de la composante sociolinguistique des enseignes nous édifiera davantage sur cette gestion in vivo du plurilinguisme urbain.

\section{Les langues, les murs et le « désordre "}

L'affichage public des langues sur les murs à l'entrée du campus universitaire de Dschang révèle une espèce de «désordre » dans la superposition des régimes linguistiques où le standard côtoie le non standard. Le bilinguisme officiel (français/anglais) de l'Etat et de l'administration universitaire s'actualise dans l'affichage au niveau des enseignes, mais on peut remarquer aussi que des codes linguistiques non officiels s'entremêlent et donnent la mesure de la dynamique des langues dans cet espace public. Même si, dans une certaine mesure, cet espace est reconnu comme étant hétérogène, instable, et dynamique, l'affichage public y est encore largement dominé par le français, comme tous les espaces francophones au Cameroun d'ailleurs. Le relevé systématique des enseignes publiques le long de ce bout de rue qui longe l'entrée du campus universitaire laisse croire a priori à une communauté anglophone ou même vernaculaire assez peu présente dans «l'espace vécu » (Boudreau et Dubois 2005:193). Nous avons recensé la disposition des langues dans les enseignes selon trois dispositions :

1. les enseignes monolingues francophones ou anglophones où n'apparaît aucune unité lexicale en anglais ou en français ;

2. les enseignes partiellement ou entièrement bilingues, avec une langue dominante ;

3. les enseignes trilingues, avec une langue dominante.

\section{Affichage monolingue}

Dans cette catégorie, nous distinguons deux types d'enseignes: les enseignes entièrement en français où n'apparaît aucune unité lexicale en anglais et les enseignes entièrement en anglais où n'apparaît aucune unité lexicale en 
français. Si les deux langues n'ont pas à chaque fois la même proportion sur le plan quantitatif, à cause justement de l'environnement qui est en majorité francophone, on note tout de même la présence des enseignes entièrement en anglais, soit parce que le propriétaire du lieu est anglophone, soit que la cible marchande est anglophone. Mais dans un cas comme dans l'autre, c'est bien plus l'origine culturelle et linguistique du commerçant qui conditionne et justifie le choix de la langue dans l'élaboration des enseignes. Sur un total de 40 affiches répertoriées dans cet espace, 16 sont entièrement en français, tant au niveau de la raison sociale (désignation générique) que de la description du commerce. On prendra juste à titre d'exemple

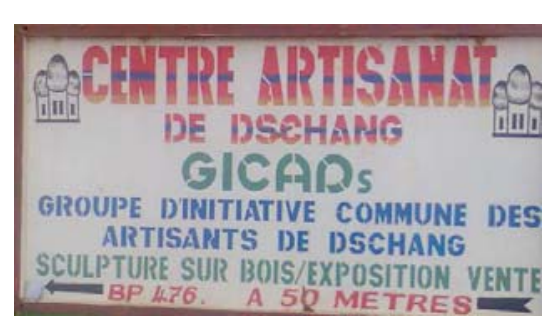

(6a)

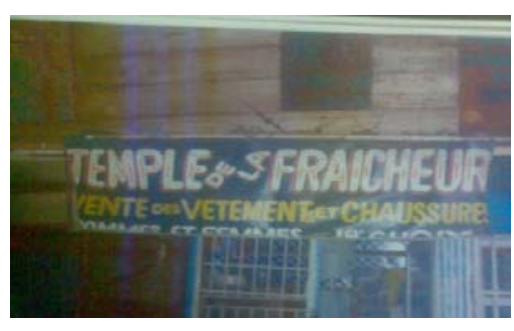

(6b)

3 des 40 enseignes seulement sont entièrement en anglais (raison sociale et description également), comme on peut l'observer dans:

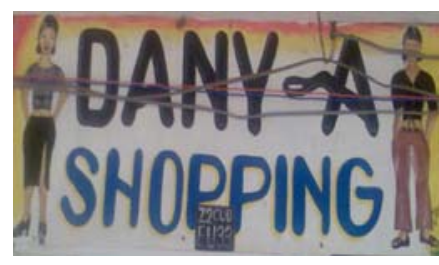

(7a)

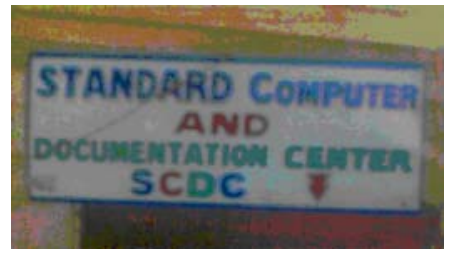

(7b)

Cette nette omniprésence du français dans l'affichage public des langues est la preuve même qu'il reste, à maints égards, la langue d'intégration dans les villes de l'espace francophone au Cameroun, comme l'ont par ailleurs montré les études sociolinguistiques récentes. On peut, à titre d'exemple, citer l'étude faite par Koenig et al. (1983) sur les pratiques et les attitudes linguistiques au sein de quelques 4850 ménages à Yaoundé, qui ont montré que 
l'hétérogénéité linguistique y est un phénomène assez frappant : les variétés vernaculaires y sont prioritairement employées dans les foyers ou entre membres d'une même ethnie, mais au regard des pratiques linguistiques extérieures aux ménages, et surtout de l'urbanisation, les auteurs concluent également à l'unification linguistique autour du français par le biais de sa véhicularisation. Tendance confirmée par Bitja’a Kody (2000:180) qui, lors d'une enquête sur les usages déclarés et la transmission inter-générationnelle des langues à Yaoundé, conclut à la « francisation galopante de la capitale camerounaise et la désuétude des langues nationales ».

En effet, si la ville est le lieu par excellence de l'unification linguistique et que l'urbanisation impose la nécessité d'une langue d'intégration à la ville, le français à Yaoundé se présente comme cette «langue véhiculaire autour de laquelle s'opère l'uniformisation linguistique de la ville et du pays » du fait de l'accroissement de son emploi dans toutes les situations informelles où l'on « assistait jadis à l'utilisation d'une langue véhiculaire locale ». Bitja'a Kody (2000:113) soutient donc que les politiques linguistiques successives du Cameroun ont largement favorisé l' « unification linguistique » autour du français, et, dans une moindre mesure, l'anglais, en leur permettant d'occuper toutes les fonctions sociolinguistiques « institutionnalisées ». Ce statut leur a conféré d'emblée « une valeur importante sur le marché linguistique », et, par conséquent, la « minoration des autres langues ».

\section{Affichage bilingue}

L'affichage bilingue est, dans l'ensemble des enseignes observées, partiel, dans la mesure où il y a une langue dominante qui est, dans la majorité des cas, le français. On peut observer ici deux types de bilinguismes : le bilinguisme officiel, celui garanti par la Constitution et l'Etat, le français et l'anglais, et un bilinguisme informel, celui où le français ou l'anglais côtoient une autre langue (étrangère ou locale).

\section{Affichage bilingue avec obligation juridique}

La Constitution camerounaise exige et garantit l'utilisation de l'anglais et du français comme langues officielles, dans les situations formelles ou officielles. Cette situation est bien présente dans l'ensemble des enseignes que nous avons observées, même si ces enseignes ne se réfèrent ou ne métérialisent pas une portion d'un espace public officiel (banques, entreprises d'envergure nationale, espaces administratifs, toponymes...). La plupart des enseignes se réfèrent aux commerces de très petite taille, et donc à des entreprises rudimentaires ayant une envergure locale ou ponctuelle (restaurants, salons de coiffure, studios photos, secrétariats bureautiques...). L'utilisation du français et de l'anglais n'est pas dictée dans ces situations par un principe 
juridique quelconque, mais par la situation de parole ou les enjeux liés au marketing.

Dans l'ensemble des situations de bilinguisme français/anglais observées, le français reste dominant, et on le retrouve très souvent dans les descriptions ou les caractérisations de la spécialisation des lieux qui s'étendent très souvent sur plusieurs phrases. L’anglais n’apparaît qu'au niveau de l'énoncé de la raison sociale (nom générique du commerce ou nom de baptême, avec plus ou moins nom générique exprimé). Il se limite très souvent à une phrase lapidaire et parfois même un mot (8a) ou syntagme (8b) :

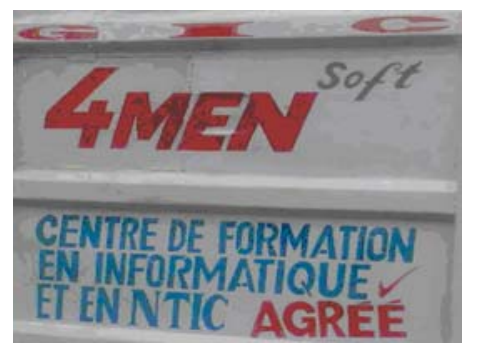

(8a)

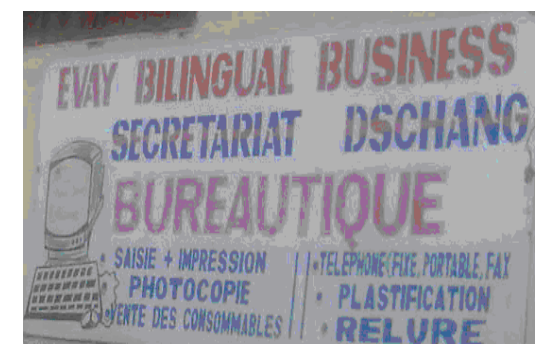

(8b)

Sur un total de 21 enseignes qui affichent un caractère bilingue, 16 se présentent de cette façon, soit 76.1 pour cent qui représentent au moins 40 pour cent de l'ensemble des enseignes. Le constat que nous faisons à ce niveau c'est qu'il n'y a aucune enseigne bilingue anglais/français, avec l'anglais dominant.

Affichage bilingue sans obligation juridique

Dans cette catégorie, nous avons observé plusieurs situations où deux langues au moins se côtoient dans une même enseigne, en dehors de celle décrite plus haut, selon la typologie suivante élaborée sur la base de nos propres observations:

\section{Anglais/chinois}

Nous avons ici un cas unique (soit $02.5 \%$ ), celui de l'enseigne des produits pharmaceutiques "TIANSHI », herboristerie chinoise de renommée internationale : 


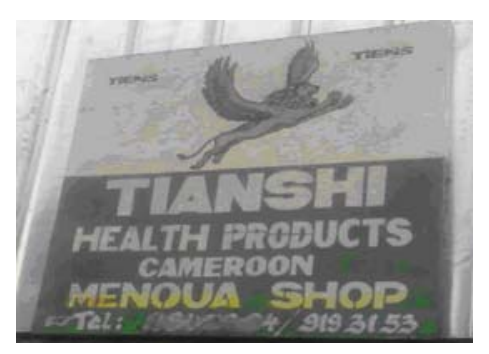

(9)

La seule présence du chinois à ce niveau réside dans l'énoncé de la raison sociale. Cette présence est sans doute liée à la mondialisation à laquelle est liée la présence économique chinoise de plus en plus forte en Afrique. La seule évocation du nom est en elle-même significative de cette nouvelle donne, mais aussi des nouveaux enjeux linguistiques dans les villes camerounaises et africaines en général. ${ }^{9}$ " TIANSHI » en fait est la traduction savante de l'expression métaphorique chinoise "le lion qui vole ", et on comprend non seulement le logo du commerce (le lion avec des ailes), mais aussi la référence aux produits de santé.

\section{Français/pidgin-english}

Nous avons ici un cas unique (2.5\%) où le français alterne avec une autre langue véhiculaire (deux véhiculaires en somme), le pidgin-english qui est très présent dans les milieux populeux au Cameroun (notamment dans les marchés, les gares routières, les ports, etc.). Mais comme dans le cas précédent, le français est dominant et l'autre langue n'apparaît que dans un syntagme ou un mot unique dans la description du commerce, et non dans l'énoncé titre qui est suffisamment mis en exergue. Il s'agit, dans le cas de cet exemple, d'un mot d'emprunt, le terme " call box ». ${ }^{10}$ Ce terme populaire est sans doute l'une des conséquences du développement et de l'essor de la téléphonie mobile au Cameroun qui a généré un nouveau vocabulaire chez les utilisateurs, comme l’a par ailleurs révélé Dassi (2003) :

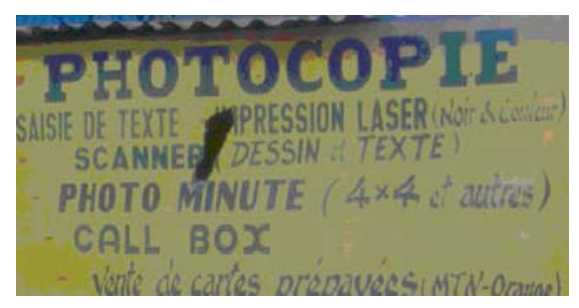

(10) 
Le terme « call box » désigne à la fois une cabine téléphonique mobile (une table, un parapluie, un ou deux téléphones mobiles) et une activité (un jeune homme ou une jeune fille assis(e)) à côté de la table) qui a généré de nombreux emplois chez des jeunes diplômés ou non en chômage au Cameroun. L'utilisation du pidgin-english atteste d'ailleurs de la force véhiculaire assez poussée de cette langue dans les mileux urbains, et surtout dans les milieux populaires.

\section{Français/argot}

Trois enseignes se présentent sous cette combinaison (7,5\%), et la présence des mots argotiques dans le français est liée sans doute à la proximité de l'université où les étudiants se sont fabriqué un jargon propre assumant une fonction cryptique certes, mais de plus en plus une fonction identitaire : ${ }^{11}$

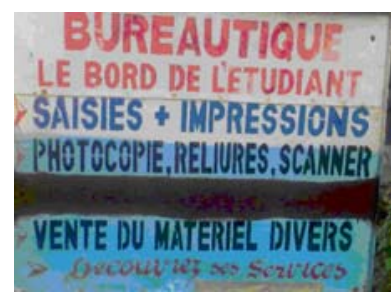

(11a)

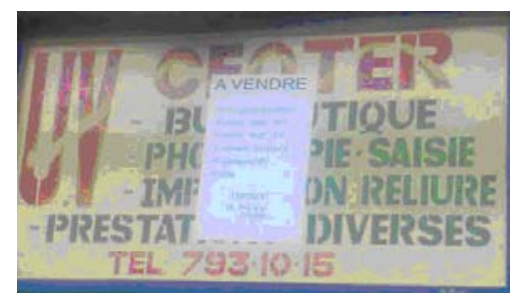

11b)

Les termes « bord » (11a) et « UV » (11b) de ces deux enseignes se réfèrent au monde estudiantin. Le premier, qui signifie "documentation », est compréhensible pour le commerce en question puisque c'est le lieu même où les étudiants photocopient divers documents qu'ils utilisent, et le second terme, qui est le sigle de " Unité de Valeur », se réfère au même univers. On comprend que pour dire les réalités du milieu, les étudiants se fabriquent un langage propre qui a une nette tendance à se populariser et à s'imposer dans l'univers des pratiques linguistiques sur le campus et dans les résidences d'étudiants.

\section{Affichage trilingue}

La seule enseigne (2,5\%) qui alterne plus de deux langues que nous avons observée est celle de ce restaurant en (1d) " CHEZ MAA-THE »., enseigne placée en bordure de route sous un poteau électrique devant un bâtiment. La façon dont elle est fabriquée (des inscriptions à la craie blanche sur un tableau en bois), son graphisme indiquent qu'il s'agit probablement d'un écrit informel. Il décrit le menu d'un restaurant invisible (situé derrière le bâtiment), sans les prix. Cette description parfois illisible est faite de manière 
générale en français, mais on y observe des noms de mets en langue camerounaise (notamment le duala avec le terme «dolé »), et en pidginenglish avec les termes « condré » et « Maa » (terme affectif et populaire désignant la maman). On ne doit pas perdre de vue le terme «THE » en français populaire (et donc argotique), qui est le diminutif de « Thérèse », le nom même du propriétaire ${ }^{12}$ qui s'avère lui aussi sur le plan publicitaire comme un argument de vente. Cette constellation des langues est certes la marque d'une insécurité langagière, mais aussi la preuve de l'hétérogéneité des pratiques induit de la «complexité » des situations.

Par son dispositif linguistique, cette affiche rappelle étrangement celle « du congélateur » décrite par Didier de Robillard (2005:136-140) dans une situation linguistique similaire à celle du Cameroun, l'Ile Maurice, et les conclusions qu'il en a tirées restent valables ici, à savoir que par son dispositif linguistique, l'affiche «ne fait d'effort ni pour inclure, ni pour exclure l'étranger : elle fait l'hypothèse que celui qui lira est [Camerounais] trilingue (s'il sait lire, il est probablement trilingue), et il n'y a aucun codage graphique particulier pour rendre le [pidgin] distinct des autres langues, donc malaisément lisible d'un "étranger » (...) son décodage repose sur une certaine connivence et sur une certaine " compétence plurilingue " » (Ibidem:140-141).

Le tableau ci-après récapitule le " poids » des langues dans l'affichage public tel que nous l'avons décrit dans cette communication. 
Tableau1 : Typologie de l'affichage public à l'entrée de l’Université de Dschang (juillet-août 2008)

\begin{tabular}{|c|c|c|}
\hline & $\begin{array}{l}\text { Nombre d'enseignes } \\
\text { recensées }\end{array}$ & $\begin{array}{l}\text { Pourcen- } \\
\text { tages }\end{array}$ \\
\hline $\begin{array}{l}\text { Affichage monolingue } \\
\text { français }\end{array}$ & 16 & $40 \%$ \\
\hline $\begin{array}{l}\text { Affichage monolingue } \\
\text { anglais }\end{array}$ & 03 & $07.5 \%$ \\
\hline $\begin{array}{l}\text { Affichage partiellement } \\
\text { bilngue, français dominant }\end{array}$ & 16 & $40 \%$ \\
\hline $\begin{array}{l}\text { Affichage partiellement } \\
\text { bilngue, anglais dominant }\end{array}$ & 0 & $0 \%$ \\
\hline $\begin{array}{l}\text { Affichage partiellement } \\
\text { bilngue,(français/ anglais) + } \\
\text { langue étrangère }\end{array}$ & 01 & $02.5 \%$ \\
\hline $\begin{array}{l}\text { Affichage partiellement } \\
\text { bilngue,(français/ anglais) } \\
\text { + argot ou pidgin-english }\end{array}$ & 03 & $07.5 \%$ \\
\hline $\begin{array}{l}\text { Affichage partiellement } \\
\text { bilngue, (français/anglais) } \\
\text { + argot ou pidgin-english } \\
\text { + langue camerounaise }\end{array}$ & 01 & $02.5 \%$ \\
\hline Total d'enseignes recensées & 40 & \\
\hline
\end{tabular}

Au regard de ce tableau, l'on peut tirer quatre grandes conclusions, à savoir :

1. que le français est majoritaire dans l'affichage public, même si l'usage normatif n’est pas généralisé dans tous les cas ;

2. que les langues camerounaises occupent une place marginale ;

3. que les parlers jeunes (argot, langues mixtes) s'accaparent une bonne portion de l'espace linguistique ;

4. que l'usage exclusif de l'anglais est marginal. 
Ce tableau, qui n'est qu'une illustration d'une situation linguistique, ne doit pas nous faire perdre de vue le fait que dans une situation de plurilinguisme comme la ville, des espaces différents peuvent avoir des configurations linguistiques différentes et qu'il ne saurait y avoir homogénéité ou stabilité tant dans les pratiques que dans les situations linguistiques. Calvet (1994:11) a d'ailleurs reconnu que dire d'une ville (ou d'un pays) qu'elle est plurilingue ne veut pas dire que tous les points le sont. Il peut y avoir des zones de monolinguisme (dans le plurilinguisme) et des zones de plurilinguisme différentes ; toujours est-il que « le plurilinguisme se manifeste là où ces monolinguismes convergent, sur la piste, les marchés, les ports, et, de façon générale, dans la ville « à laquelle aboutissent les pistes et où se trouvent les marchés et les ports »".

Cette recherche menée à différents points ou lieux publics dans la ville de Dschang (ou d'autres villes camerounaises) peut produire des résultats radicalement ou relativement différents. Chaque lieu produit et conditionne ses usages, ses langues en raison des complexités qui caractérisent les espaces sociaux. L'affichage public des langues dans les espaces publics ne procède d'aucune logique « officielle » contrôlable, chaque espace régule et élabore ses propres politiques linguistiques in vivo, et il n'y a aucune pratique de langue qui soit prédictible ou " décontextualisée » (De Robillard, op. cit.). A en croire Feussi (2006), « si la langue reste une construction sociale, la démarche pour y accéder ne peut occulter la contextualisation et ses conséquences ». Le rôle ou l'influence de la ville sur les langues ou l'environnement linguistique est, de ce point de vue, déterminant, voire fondamental. La ville, en effet, n'existe pas sans le pays dans lequel elle se trouve : l'histoire politique, sociale et démographique rejaillit sur elle et sur ses langues (Calvet 2000:11).

\section{Et les langues nationales?}

L'observation du tableau I amène à se poser des questions sur le rôle, le poids et la place même des langues nationales dans l'administration publique des langues dans des situations de plurilinguisme au Cameroun. Si la Constitution accorde une place prépondérante au français et à l'anglais, elle est assez muette sur le sort de près de 245 langues nationales qui les côtoient et qui pourtant portent un ancrage identitaire très poussé. ${ }^{13}$ Comment comprendre en effet que le yemba, ${ }^{14}$ qui est pourtant si présent dans la communication orale à Dschang (notamment dans les marchés, les familles, l'église et les cérémonies traditionnelles), soit si absent sur les murs de la ville ? On comprend donc qu'en dehors des langues officielles et de certains véhiculaires bien répandus comme le pidgin-english, le camfranglais chez les jeunes, les langues nationales ne relèvent pas encore de la sphère du 
public. Elles sont réduites à un usage oral ou populaire dans des situations marginales ou quasi privées. La seule enseigne où nous retrouvons une langue nationale (le duala) dans l'ensemble des enseignes que nous avons observées à l'entrée du campus universitaire est celle d'un restaurant traditionnel, banal et assez rudimentaire, et, pour preuve, l'enseigne elle-même est écrite à la main, à la craie blanche et donc ne s'inscrit pas dans la durée. L'utilisation de ces langues varie en fonction des menus et il est aisé de croire que ces langues sur les enseignes ont un fonctionnement paradigmatique, c'est-àdire ne servent que dans des situations d'emprunts ou de substitution lexicale, là où le français ou l'anglais sont incapables d'exprimer la réalité désignée. Leur emploi est essentiellement provisoire ou ponctuel dans des enseignes qui n'ont elles-mêmes aucun caractère « public » ou officiel ».

C'est peut-être bien la preuve que la politique linguistique officielle exerce encore une influence considérable sur la tradition de l'écrit et la gestion du plurilinguisme camerounais. Beaucoup de langues ne sont pas écrites ou ne sont pas enseignées dans nos écoles certes, mais elles jouent un rôle non négligeable dans l'affirmation des identités individuelles et collectives, et le fait qu'elles soient exclues constitue une perte et une déperdition linguistique énorme. Cette politique linguistique a, paradoxalement, réservé aux langues camerounaises un statut "négligeable de marqueur linguistique d'appartenance à une communauté », confinées à un usage oral, familial et ethnique, et, par conséquent, la "valeur marchande » qu'y attachent leurs locuteurs ne peut être que négligeable (Bitja'a Kody 2000:113). Tout en réfutant la conception d'un environnement urbain « tout en français ", Harter (2005:94) reconnaît que, même si elles sont « minorées officiellement», les langues camerounaises conservent « des fonctions qui sont loin d'être négligeables », car, si la ville unifie pour des besoins d'efficacité véhiculaire, elle ne peut néanmoins « réduire un besoin identitaire qui s'exprime au travers des langues camerounaises ».

La valorisation annoncée de ces langues au Cameroun, et notamment la création des centres de langues et cultures camerounaises dans les universités et même l'ouverture d'une filière de langues camerounaises à l'Ecole Normale Supérieure, leur enseignement dans les écoles, collèges et lycées seraient un palliatif énorme à cette situation et contribueraient à l'affirmation d'une identité proprement camerounaise que les langues issues de la colonisation sont loin d'exprimer dans leur totalité de nos jours. Même si «l'autochtonie » ou "l'insularité » linguistiques ne sont plus envisageables dans un contexte d'ouverture économique et culturelle mondiale, on ne saurait envisager, comme le dit Foued Laroussi (2003:4), le développement « durable » d'un pays dans une langue étrangère, celle qui n'est pas assumée par une bonne partie de ses locuteurs. L'action de ceux-ci à eux seuls ne peut 
malheureusement suffire pour définir une politique linguistique. Le rôle de l'Etat n'est pas à négliger dans cette "action » qui consiste à définir une langue non seulement comme status, mais aussi comme corpus (Chaudenson 1991:50). ${ }^{15}$

\section{Conclusion}

Au vu de ce qui précède, on peut dire qu'on ne peut, dans le cadre d'une sociolinguistique urbaine, se départir de la « théorie sociologique de la ville ${ }^{16}$ qui associe à la description des langues le terrain sur lequel elles vivent. Le rôle ou l'influence de la ville sur les langues ou l'environnement linguistique est déterminant, voire fondamental. La ville, à en croire Calvet (2000:11), n'existe pas sans le pays dans lequel elle se trouve : l'histoire politique, sociale et démographique rejaillit sur elle et sur ses langues. On comprend que la ville ou les espaces de ville peuvent déterminer eux-mêmes leurs propres politiques linguistiques. Chacun de ses espaces, de ses lieux gère ses langues ou ses codes, gouverne et administre ses propres pratiques dans une espèce de " désordre » que commandent l’hétérogénéité des situations et la «floraison des compétences diverses nécessaires à la communication linguistique " (Calvet 2000:19). L'étude des observables menée ci-dessus montre que ce sont autant les murs qui font les langues que l'inverse : le fait d'afficher des discours sur les murs, dans les lieux symboliques affirme l'existence de langues, de statuts, d'identités ou permet de les construire, en même temps que ceux-ci contribuent à définir les langues, les groupes, les statuts des individus. Au lieu de ne trouver que des langues dans l'homogénéité, la décontextualité, la stabilité, on s'aperçoit donc qu'elles peuvent fonctionner dans l'hétérogénéité, l'instabilité, la contextualité (de Robillard 2005:146), bref, le « désordre ».

De ce point de vue, on peut considérer la ville comme un acteur indispensable de la politique linguistique puisqu'elle « intervient sur les situations ». Nombreuses sont en effet, les villes dans lesquelles c'est la pratique sociale des locuteurs qui dessine l'avenir linguistique de la ville et souvent du pays. Ce mécanisme de tri urbain emprunté à la sociologie urbaine de l'Ecole de Chicago (Grafmeyer op. cit. 51) est donc, selon Calvet (2000:26), ce qui définit le mieux «l'action de la ville sur les situations linguistiques » : elle pousse à faire le tri, à promouvoir certaines langues à fonction véhiculaire, à en limiter d'autres aux fonctions grégaires, voire à les abandonner.

\section{Notes}

1. Colloque organisé par le CALS/CPST Université de Toulouse le Mirail, dont les actes ont paru en juillet 2003, sous la direction de Pierre Marillaud et Robert Gauthier. 
2. Dans la conception d'une linguistique dite « affranchie » (Gasquet-Cyrus 2008), Louis-Jean Calvet préfère ce terme flou et générique de « gens » pour désigner ce que les autres sociolinguistes appellent « locuteurs », " sujets parlants », « informateurs » ou " enquêtés ».

3. Calvet cite, comme bel exemple de ce désordre des pratiques et des différentes façons de le traiter, une illustration faite par Didier de Robillard (2005) sur une affiche manuscrite accolée sur le congélateur d'une épicerie de l'Île Maurice et donnant une liste des produits et de leur prix.

4. Une école régionale à vocation agricole.

5. Auxquels on ajoute l'ancienne Université de Yaoundé éclatée en deux universités, Yaoundé I et Yaoundé II.

6. Les estimations sont de Ngamini (2003).

7. Il convient d'ajouter que le Département de la Menoua dont la ville de Dschang est le chef-lieu partage sa frontière ouest avec le Département de Lebialem (moins de $50 \mathrm{~km}$ ) dans la province du sud-ouest anglophone, et est aussi à une centaine de kilomètres de la province du nord-ouest anglophone également. On comprend la position de la ville comme carrefour de langues et d'intégration linguistique.

8. Expression en pidgin-english qui désigne au Cameroun les points d'appels payants et donc des cabines téléphoniques mobiles (ou amovibles).

9. On peut, à cet égard, observer la percée du chinois dans le système éducatif camerounais comme langue étrangère, à côté de l'italien, de l'espagnol et de l'allemand. La toute nouvelle université de Maroua par exemple propose, dans la filière « langues étrangères ", le chinois comme cycle de formation.

10. Un mot ou parfois en deux, selon les cas. Il n'y a pas en fait une graphie unique !

11. Voir aussi Tandia et Tsofack (2004 et 2006).

12. Et non le thé, qui est l'un des produits consommés dans ce restaurant. L'homologie est bien perceptible et participe du jeu publicitaire.

13. Même si ces derniers temps des efforts louables sont faits pour la vulgarisation et l'intégration des langues maternelles dans les écoles, les collèges et les universités.

14. C'est la langue nationale dominante dans la ville de Dschang et le département de la Menoua en particulier. Il est la langue maternelle de nombreux locuteurs de la ville et sert de véhiculaire dans plusieurs situations d'usage dans la ville et dans les villages voisins.

15. Chaudenson entend donc par status tout ce qui relève aussi bien du « statut » proprement dit (reconnaissances officielles et légales, usages institutionnels hors de l'éducation...) que des "fonctions " (éducation et moyens de communication de masse) ou enfin du « statut économique » (capacité de la langue à assurer la réussite professionnelle de ceux qui la parlent). Status, de manière générale, désigne à la fois le statut (langue officielle, unique ou non, nationale, etc.), les emplois (officiel, administratif, juridique, etc.) et les fonctions. Au corpus sont associés quatre grands ensembles que sont les 
modes d'appropriation, la véhicularisation et/ou vernacularisation, les types de compétences, les productions et consommations langagières.

16. Mise en valeur par les sociologues membres de l'équipe de l'«école de Chicago » qui ont développé l'idée de la ville comme un « laboratoire social » (cf. A. Coulon, L’Ecole de Chicago, Paris, PUF, 1992).

\section{Bibliographie}

Barthes, R., 1985, « Sémiologie et urbanisme », in L’Aventure sémiologique, Paris, Seuil, pp. 261-271.

Bitja'a Kody, D. Z., 2000, « Vitalité des langues à Yaoundé : le choix conscient », in Calvet, L-J et Moussirou-Mouyama, A., éds., Le plurilinguisme urbain, Actes du Colloque International de Libreville, ENS Libreville, 25-29 septembre 2000, Collection Langues et Développement, Paris, Didier Erudition et Institut de la Francophonie, pp. 163-182.

Blanchet, P., 2007, « Quels « linguistes » parlent de quoi, à qui, quand, comment et pourquoi? Pour un débat épistémologique sur l'étude des phénomènes linguistiques ", in Carnets d'atelier de sociolinguistique, ${ }^{\circ} 1$, Paris, L'Harmattan.

Boudreau, A. et Dubois, L., 2005, « L’Affichage à Moncton : masque ou miroir ? », in Signalétiques et signalisations linguistiques et langagières des espaces de ville. Actes de la 4 ème Journée internationale de Sociolinguistique urbaine, Moncton, septembre 2005, Revue de l'Université de Moncton, n 1 , vol. 36, Moncton, pp. 183-218.

Bulot, T., 2001, « Ségrégation et urbanisation linguistique : l’altérité urbaine définie ou «l'étranger est une personne » », in Diversité langues (en ligne), Vol.VI Québec, Téléuniversité, 22 p. [http://www.teluq.uquebec.ca/diverscite/ entree.htm]

Bulot, T., 2002, «La double articulation de la spatialité urbaine : «espaces urbanisée » et "lieux de ville » en sociolinguistique », in Margeslinguistiques, $\mathrm{n}^{\circ} 3$, [http://www.marges-linguistiques.com], Saint-Chamas, M.L.M.S Editeur, pp. 91-105.

Bulot, T. et Veschambre, V., dirs., 2006, Mots, traces et marques. Dimensions spatiale et linguistique de la mémoire urbaine, Paris, L'Harmattan.

Calvet, L.-J., 1993, La sociolinguistique, Paris, PUF, coll. « Que sais-je ? ».

Calvet, L.-J., 1994, Les voix de la ville. Introduction à la sociolinguistique urbaine, Paris, Payot.

Calvet, L.-J., 2000, « La ville et la gestion in vivo des situations linguistiques », in Calvet, L.-J et Moussirou-Mouyama, A., éds., Le plurilinguisme urbain, Actes $d u$ Colloque International de Libreville, ENS Libreville, 25-29 septembre 2000, Collection Langues et Développement, Paris, Didier Erudition et Institut de la Francophonie, pp. 11-30.

Calvet, L-J., 2007, « Pour une linguistique du désordre et de la complexité », in Carnets d'atelier de sociolinguistique, ${ }^{\circ}{ }^{\circ}$, Paris, L’Harmattan, pp.3-71. 
Chaudenson, R., 1991, La francophonie : représentations, réalités, perspectives, Université de Provence, Institut d'Etudes créoles et francophones et Didier Erudition

Coulon, A., 1992, L'Ecole de Chicago, Paris, PUF, « Que sais-je ? ».

Dassi, E., 2003, « Question de sémantique : de la néologie autour de la téléphonie mobile », Sudlangues, $n^{\circ} 2$ (en ligne), Dakar, UCAD, pp. 21-38.

Feussi, V., 2006, « Une construction du français à Douala », Thèse de Doctorat Nouveau Régime, Université François de Rabelais de Tours (France), inédit.

Gasquet-Cyrus, M., 2008, « « Ni dieu, ni maître - mais des gens qui parlent »: la linguistique affranchie de Louis-Jean Calvet », in Moussirou-Mouyama, A., éd, Les boîtes noires de Louis-Jean Calvet, Paris, Ecriture, pp. 79-98.

Grafmeyer, Y. et Joseph, I., 1990, L'École de Chicago. Naissance de l'écologie urbaine, Paris, Aubier.

Harter, A.F., 2005, « Cultures de l’oral et de l'écrit à Yaoundé », in Glottopol, $n^{\circ} 5$ (en ligne), [http://www.univ-rouen.fr/dyalang/glottopol]

Koening, E., Chia, E., POVEY, J., éds., 1983, A Sociolinguistic Profile of Urban Centers in Cameroon, Los Angeles, Crossroads Press.

Laroussi. F., 2003 : « Présentation », in Glottopol, n¹ (en ligne). [www.univrouen/dyalang/glottopol]

Mondala, L., 2000, Décrire la ville. La construction des savoirs urbains dans l'interaction et dans le texte, Paris, Ed. Economica.

Moreau, M.L., 1997, La sociolinguistique : concepts de base, Bruxelles, Mardaga.

Ngamini, N.A., 2003, « Activité des garages de réparation et de dégradation du cadre urbain », Mémoire de Maîtrise, Université de Dschang.

Ripoll, F., 2006, « Réflexions sur les rapports entre marquage et appropriation de l'espace ", in Mots, traces et marques. Dimensions spatiale et linguistique de la mémoire urbaine, Paris, L’Harmattan, pp. 15-36.

Robillard, D. de, 2001, « Peut-on construire des « fais linguistiques » comme chaotiques ? Quelques éléments de réflexion pour amorcer le débat », in Marges-linguistiques, $\mathrm{n}^{\circ} 1$, pp. 163-204).

Robillard, D. de, 2005, «Quand les langues font le mur lorsque les murs font peut-être les langues : Mobilis in mobile, ou la linguistique de Nemo », in Signalétiques et signalisations linguistiques et langagières des espaces de ville. Actes de la $4^{\text {ème }}$ Journée internationale de Sociolinguistique urbaine, Moncton, septembre 2005, Revue de l'Université de Moncton, $\mathrm{n}^{\circ} 1$, vol. 36, Moncton, pp. 129-154.

Tandia, J.J.R. et Tsofack, J. B., 2004 « L’argot estudiantin dans le campus universitaire de Dschang ", in FOSSO, dir., Dynamique du français au Cameroun : problèmes sociolinguistiques et stylistiques, aspects didactiques et glottopolitiques,, Yaoundé, Presses universitaires d’Afrique, 2004, pp.123-144.

Tandia, J.J.R, et Tsofack, J. B, 2006, « Discours estudiantins et manifestations argotiques : parole malheureuse ou parole vengeresse ? », in Actes du XXVIème Colloque international d'Albi « langages et signification “ les “ discours de la vengeance », Toulouse, C.A.L.S / C.P.S.T, pp. 61-70. 
